\title{
GB20 Pharmacopuncture As a Potential Method for Brain Drug Delivery via the Perivascular Spaces
}

\author{
Oxana Semyachkina-Glushkovskayaa, ${ }^{1,2,}$, Inna Blokhina ${ }^{2}$, Nikita Navolokin ${ }^{2,3}$, Arina Evsukova ${ }^{2}$, \\ Georgy Karandin ${ }^{2}$, Alexander Khorovodov², Andrey Terskov'2, Ivan Fedosov' ${ }^{2}$, Alexander Dubrovsky², \\ Maria Tzoy², Vasily Ageev ${ }^{2}$, Ilana Agranovich ${ }^{2}$, Valeria Telnova ${ }^{2}$, Anna Tsven ${ }^{2}$, Jürgen Kurths $s^{1,2,4}$ \\ ${ }^{1}$ Department of Physics, Humboldt University, Berlin, Germany \\ ${ }^{2}$ Department of Biology, Saratov State University, Saratov, Russia \\ ${ }^{3}$ Department of Anatomy, Saratov Medical State University, Saratov, Russia \\ ${ }^{4}$ Potsdam Institute for Climate Impact Research, Potsdam, Germany
}

Received August 16, 2021

Revised December 10, 2021

Accepted January 30, 2022

Correspondence to Oxana SemyachkinaGlushkovskaya

Department of Physics, Humboldt University, Berlin, Germany

E-mail glushkovskaya@mail.ru
Background: The development of new methods of drug brain delivery is a crucial step for the effective therapy of the brain diseases. Pharma- and acupuncture are the forms of alternative therapy of the brain pathology, including an increase in the permeability of blood-brain barrier. However, the mechanisms of pharma- and acupuncture-mediated effects on the brain physiology remain not fully understood.

Results: This pilot study on healthy mice clearly demonstrates the Evans Blue spreading in the mouse head and in the brain via the perivascular spaces (PVSs) of the trigeminal structure and the cribriform plate after the dye injection into the Feng Chi point (Galbladder 20, GB20).

Conclusion: These results suggest that pharmacopuncture at GB20 can be a perspective method for brain drug delivery via PVSs.

Keywords: Pharmacopuncture, The Feng Chi point, Perivascular spaces, Delivery into the brain

\section{INTRODUCTION}

Pharmacopuncture and acupuncture are safety and alternative approaches for therapy of diseases of the central nervous system (CNS) [1,2], such as stroke [3], Alzheimer's $[4,5]$ and Parkinson's [6,7] diseases, epilepsy [8], Belly's palsy [9], and headache $[10,11]$. There are intrigue results demonstrating glioma therapy using the pharmacopuncture at $\mathrm{Zu}$ San Li (ST 36) [12], delivery of choroid plexus cells into the brain via pharmacopuncture at ST36 [5] and electroacupuncture stimulation of transient enhancement of the permeability of blood-brain barrier (BBB) [13]. However, despite the fact that pharmacopuncture and acupuncture have been used in Oriental Medicine over 2000 years [14], the mechanisms underlying pharmacopuncture and acupuncture-mediated effects on the brain physiology remain poor understood.

The first study of mechanism of action of acupuncture began around 1950 from Peking University $[2,15]$. They demonstrated antinociceptive effects of acupuncture via stimulation of endogenous opioid system $[2,15]$. Later, it was shown that acupuncture at the Feng Chi (Galbladder 20, GB 20) increases the cerebral blood flow (CBF) [16]. Two independent groups demonstrated the effects of acupuncture at GB20 on $\mathrm{CO} 2$ reactivity in the basilar artery and the middle cerebral artery during hypocapnia in healthy participants $[17,18]$. Thus, these pilot studies demonstrate that acupuncture at GB20 can affect the cerebral circulation. However, this is a debated and not widely accepted view.

There is evidence that pharmacopuncture and acupuncture can modulate the drainage system of the brain that might be a crucial mechanism of therapeutic effects of this procedure for the CNS, including headache. Nowadays, the drainage system of the brain is considered as a novel target for intervention of neurological diseases $[19,20]$. The acupuncture causes therapeutic effects on the injury of outflow of the cerebrospinal fluid (CSF) and drainage system of the brain associated with the development of migraine [21-25]. The cranial circulation, both extracerebral and cerebral, is innervated by fibers from the trigeminal nerve. This system 
is known as the trigeminovascular system. Migraine occurs from the large venous sinuses and the dura mater, which are pain-sensitive and are innervated by branches of the ophthalmic division of the trigeminal nerve [21]. The reason of trigeminal neuralgia is spontaneous intracranial arterial dilation or intracranial hypotension, which results from accumulation of CSF in the dura due to the injury of CSF outflow caused by the dural or cranial nerve abnormalities, and subarachoid hemorrhages [22-25]. A number of studies have shown that acupuncture, especially GB 20 is an effective treatment for certain types of headache $[1,10,11]$. The pharmacopuncture and acupuncture-mediated modulation of drainage system of the brain and improving of CSF outflow can be a new mechanism explanting antinociceptive effects of GB20 stimulation. However, there are no studies demonstrating the effects of pharmacopuncture and acupuncture at GB20 on drainage system of the brain. In this pilot study, we aimed to analyze the connective bridge between pharmacopuncture at GB20 and the route to the fluid system of the brain.

\section{MATERIALS AND METHODS}

\section{Subjects and groups}

Male BALB/c mice (20-25 g) were used in all experiments. The animals were housed under standard laboratory conditions, with access to food and water, ad libitum. All procedures were performed in accordance with the "Guide for the Care and Use of Laboratory Animals". The experimental protocols were approved by the Local Bioethics Commission of the Saratov State University (Protocol No. 7). The experiments included the following groups: (1) the study of Evans Blue spreading in the mouse head $30 \mathrm{~min}$ after the dye injection at GB20, $n=7$; (2) the study of Evans Blue spreading in the mouse head 3 hours after the dye injection at GB20, $\mathrm{n}=7$; the study of Evans Blue spreading in the mouse brain 3 hours after the dye injection at GB20, $n=7$.

\section{In vivo monitoring of Evans Blue distribution in the mouse head and brain}

The Hamilton syringe ( $25 \mu$ l, Hamilton Co, Reno, Nevada, US) with the needle (25 Gauge) was mounted onto the injection pump using the stereotactic manipulator (Stoelting, New York, US). The $5 \mu$ lof Evans Blue dye (Sigma Chemical Co., St. Louis, Missouri, $1 \%$ solution in physiological $0.9 \%$ saline) at a rate of $1 \mu \mathrm{l} / \mathrm{min}$ was injected into GB20 under inhalation anesthesia ( $1 \%$ isoflurane at $1 \mathrm{~L} / \mathrm{min} \mathrm{N}_{2} \mathrm{O} / \mathrm{O}_{2}$ - 70/30 ratio). Pharmacopuncture injection (PhI) was performed during $5 \mathrm{~min}$ and then the needle was removed at a rate of $0.5 \mathrm{~mm} / \mathrm{min}$ to prevent the reflux of Evans Blue. The optical monitoring of the distribution of Evans Blue in the mouse head and brain was performed during $30 \mathrm{~min}$ and 3 hours after the injection of tracer into GB20. For visualization of blood vessels, fluorescein isothiocyanate (FITC)-dextran $70 \mathrm{kDa}(1 \mathrm{mg} / 25 \mathrm{~g}$ mouse, $0.5 \%$ solution in saline, SigmaAldrich) was injected into the tail vein.

The imaging was performed using homemade optical instrument based on monochrome camera acA2040-90um (Basler AG, Germany) and $50 \mathrm{~mm} \mathrm{2,8} \mathrm{C-mount} \mathrm{CCTV}$ objective lens (Tamron, Japan) The lens was attached to the camera with $15 \mathrm{~mm}$ extension tube to ensure macro imaging with 23.3 to $31.8 \mathrm{~mm}$ field of view depending of the lens focusing ring adjustment. The lens was mounted on vertical manual translation stage (Standa, Lituania) above a Petri dish where samples were submerged in buffer solution. Top surface of each sample was covered with $25 \times 50 \times 0.17 \mathrm{~mm}$ cover glass. Slider with filter sets $(49019,49002$, Chroma Technology, USA) was placed just below objective lens. Each filter set was illuminated with homemade condensers with

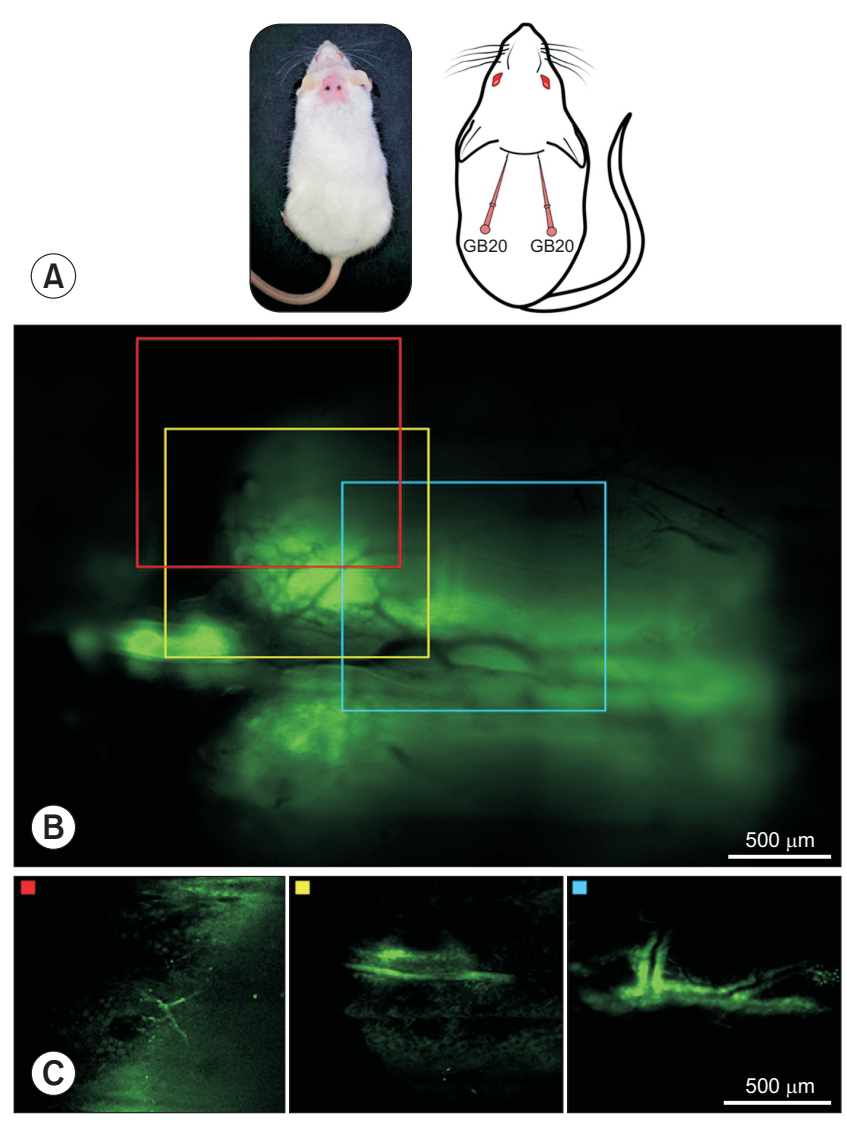

Fig. 1. Real time confocal monitoring of Evans Blue distribution in the mouse head after the dye injection at GB20: (A) the schematic illustration of the injection of Evans Blue into GB20; (B) the fluorescence from Evans Blue in the dorsal part of the mouse head $30 \mathrm{~min}$ after the dye injection into GB20; (C) the region of interest (ROI) demonstrating the perivascular pathway of dye spreading from GB20. Images are representative of $n=7$ mice. 
1W LEDs (635 nm for 49019; and $460 \mathrm{~nm}$ for 49002) to ensure uniform illumination over camera field of view. Led illuminators were synchronized with camera "fire" output.

Camera resolution of $2048 \times 2048$ pixels at 12 bit grayscale. Images were acquired in dark room at constant exposure time of $200 \mathrm{~ms}$ and other settings kept unchanged for all samples. Image acquisition and processing were performed with custom software developed using N I Vision and LabVIEW software (National Instruments, USA) and FIJI open source image processing package [26]. Image processing procedures were identical for each pair of images (control and laser treated sample) for each channel to ensure accurate comparison of fluorescence intensity.

\section{Ex vivo analysis of Evans Blue distribution in the mouse head and in the brain}

For ex vivo analysis of Evans Blue distribution in the mouse head and in the brain, we used histological and confocal analysis. The $5 \mu \mathrm{l}$ of Evans Blue dye (Sigma Chemical Co., St. Louis, Missouri, $1 \%$ solution in physiological $0.9 \%$ saline) at a rate of $1 \mu \mathrm{l} / \mathrm{min}$ was injected in GB20. Three hours after the injection of tracer into GB20, head was sacrificed using rodents guillotine (Harvard apparatus, Holliston, Massachusetts, US). Afterward, for decalcification of the bones, the heads was placed for a week at $4{ }^{\circ} \mathrm{C}$ in $10 \%$ ethylenediaminetetraacetic acid disodium salt dihydrate (EDTA-2Na), $100 \mathrm{mM}$ Tris base, $\mathrm{pH}$ 7.0, in a $2 \mathrm{ml}$ tube. Buffer was changed every other day. Next, samples were removed and fixed in $4 \%$ buffered paraformaldehyde. Paraformaldehyde-fixed specimens of head were embedded in paraffin, sectioned $(10 \mu \mathrm{m})$ and stained with hematoxylin and eosin. Histological sections were evaluated by light microscopy using the digital image analysis system Mikrovizor medical $\mu$ Vizo-103 (LOMO, Russia) with a magnification of 246. The confocal images of fluorescent signal from Evans Blue (excitation, 635 nm; emission, 670-800 $\mathrm{nm}$ ) in the same samples of mouse head, including the brain, were acquired using Nikon A1R confocal microscope with a $10 \times 0.45$ dry lens (Nikon Corp., Japan). $\mathrm{N}=7$ in each group.

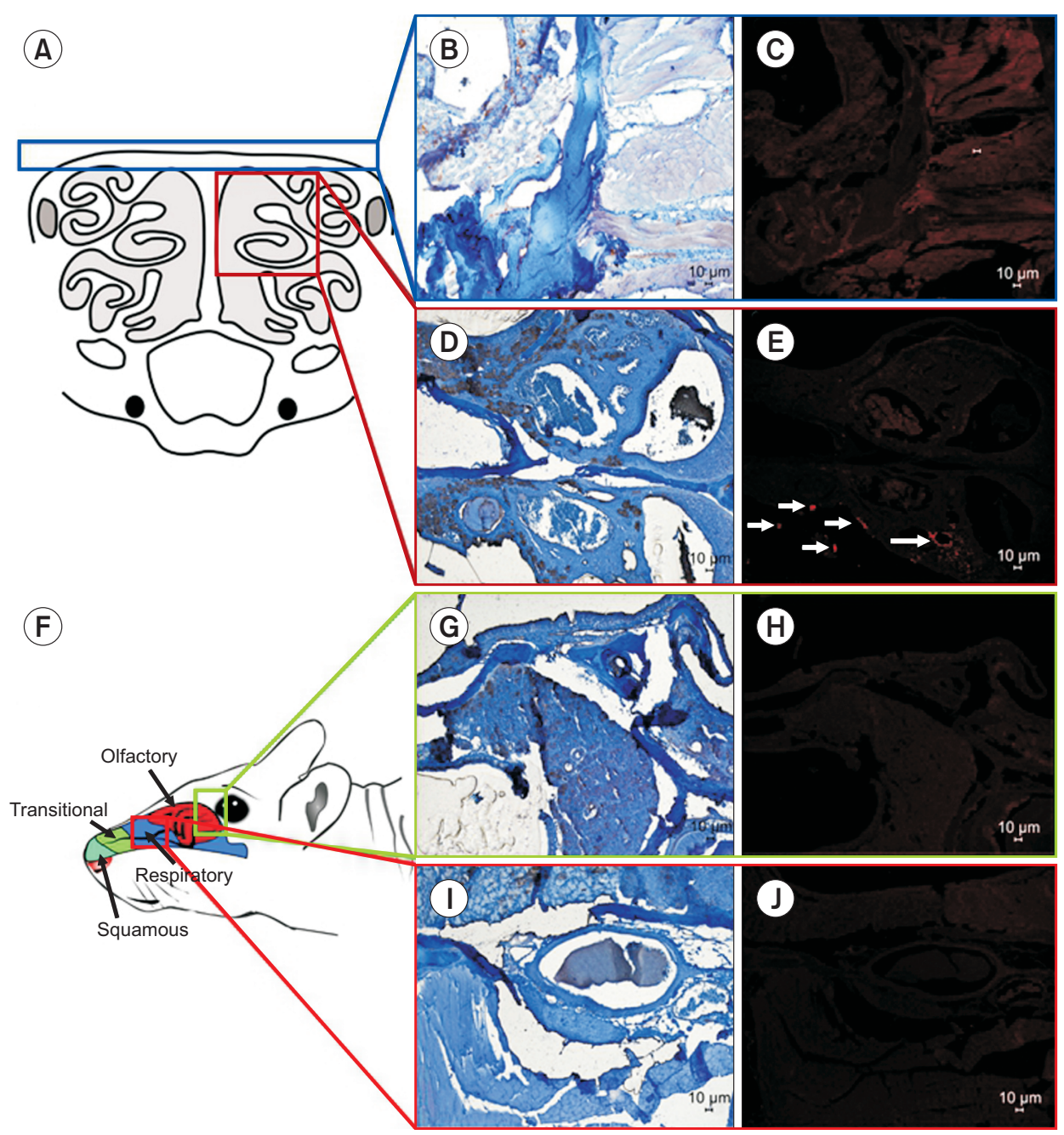

Fig. 2. The histological and confocal analysis of Evans Blue distribution in the mouse head $3 \mathrm{~h}$ after the dye injection at GB20. $(A, F)$ - the schematic illustration of ROI for the histological (left column) and the confocal (right column) analysis of presence of Evans Blue (red fluorescence, arrowed) in: $(B, C)$ the epaxial musculature; $(\mathrm{D}, \mathrm{E})$ the cribriform plate; $(G, H)$ in the ocular part of nasolacrimal duct; $(\mathrm{I}, \mathrm{J})$ in the Bowman's glands. Images are representative of $\mathrm{n}=7$ mice, scale bars with a magnification of 246. 


\section{RESULTS}

\section{Short-term Evans Blue spreading in the mouse head with $\mathrm{ACl}$}

In the first step, we analyzed the distribution of Evans Blue in the mouse head 30 min after the dye injection into GB 20 . The GB20 is located at the top of the sternocleidomastoid muscle, which runs from the back of the head down to the front of the shoulders at the clavicle (Fig. 1A). Real time confocal monitoring revealed the fast spreading of Evans Blue in the dorsal part of mouse head (Fig. 1B). Evans Blue diffused along the perivascular spaces (PVSs) in the trigeminal vasculature and in the olfactory region (Fig. 1C). Thus, the fast diffusion of Evans Blue after its injection into GB20 is observed predominately through the trigeminovascular system and the olfactory structures of the mouse head.

\section{Long-term Evans Blue spreading in the mouse head with $\mathrm{ACl}$}

In the next study, we analyzed Evans Blue diffusion 3 hours after the dye injection at GB20 using the histological analysis and the confocal imaging. Fig. 2 demonstrates the presence of Evans Blue in the tested tissues. We found the fluorescence from Evans Blue in the cribriform plate around the olfactory nerves but not in the Bowman's glands (Fig. 2A, D, E, I, J).

There was no Evans Blue in the nasolacrimal duct, which is supported to be the main route of CSF outflow in rodents (Fig. 2G, H) [27]. We also did not find Evans Blue in the epaxial musculature (Fig. 2B, C). Thus, these data suggest posterior direction of dye spearing in the mouse head with deep
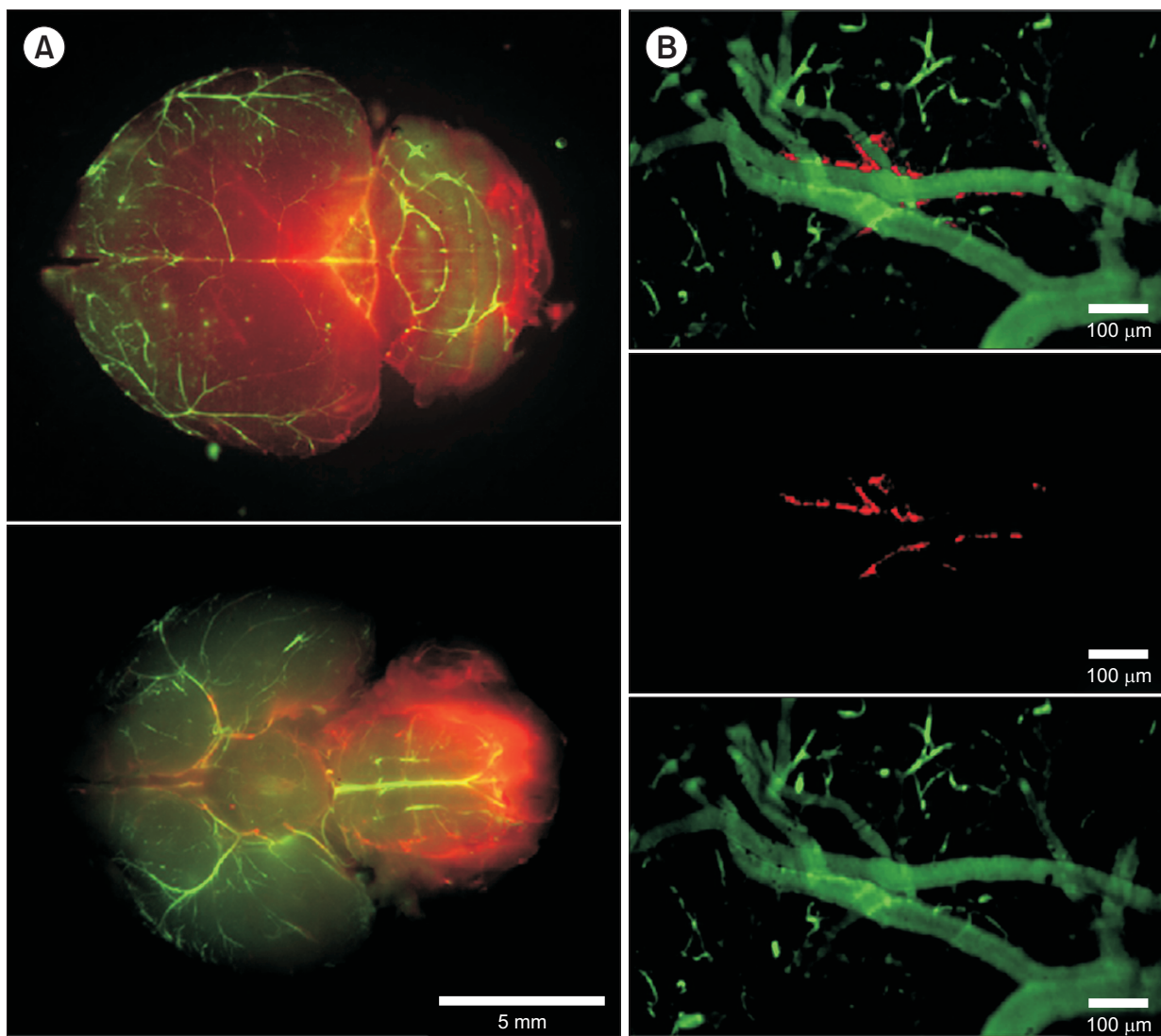

(C)

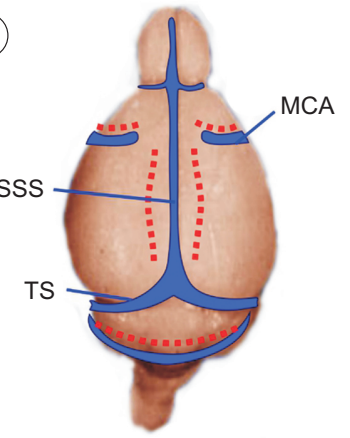

(D)

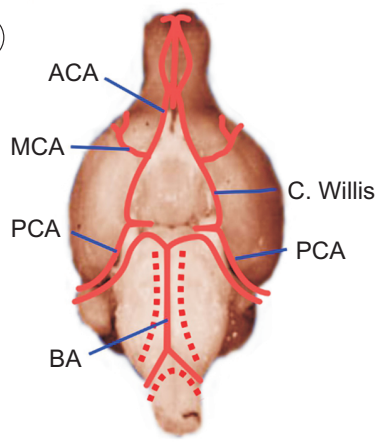

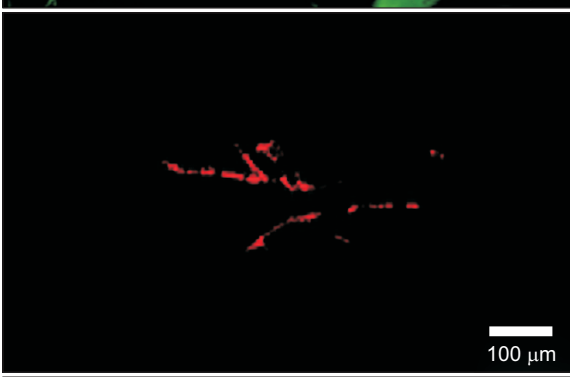

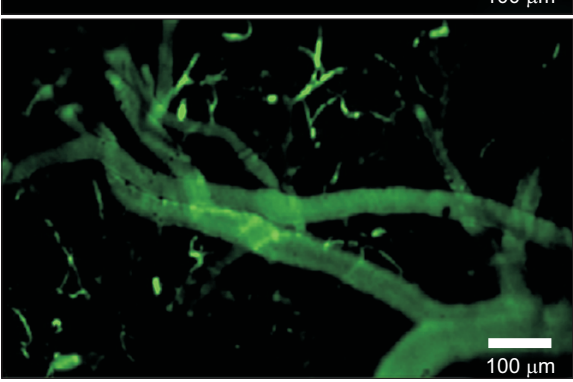

E

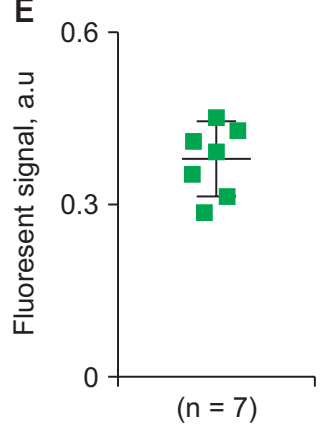

Fig. 3. Perivascular spreading of Evans Blue in the mouse brain after the dye injection at GB20. (A) Representative images of distribution of Evans Blue on the dorsal and ventral surfaces of the brain 3 hours after the dye injection at GB20; (B) The perivascular pathway of Evans Blue spreading in the brain; $(\mathrm{C}$, D) the schematic illustration of Evans Blue distribution along the cerebral veins and arteries, respectively. $\mathrm{MCA}=$ Middle cerebral artery; $\mathrm{C}$. Willis = Circle of Willis; PCA = Posterior cerebral artery; $\mathrm{BA}=$ Basilar artery; SSS = Superior sagittal sinus; TS = Transverse sinus. Red color, Evans Blue; green color, FITC-dextran; (E) The intensity of the fluorescent signal from Evans Blue in each mouse. 
penetration through the cribriform plate.

\section{Long-term Evans Blue spreading in the mouse brain with $\mathrm{ACl}$}

In the further step, we studied Evans Blue spreading in the brain 3 hours after the dye injection into GB20. Fig. 3 illustrates representative confocal images of distribution of Evans Blue along the main cerebral venous sinuses, such as the Superior sagittal sinus and the Transverse sinus as well as along the main cerebral arteries, including the Middle cerebral artery, the Circle of Willis, the Posterior cerebral artery, the Basilar artery. The mice demonstrated different presence of Evans Blue in PVSs of the brain (Fig. 3E). However, overall the group the Evans Blue distribution in the brain was homogeneous.

\section{DISCUSSION}

The pharmacopuncture and acupuncture is a form of alternative therapy of diseases of the CNS [1,2]. The delivery of different medications into the brain bypassing the $\mathrm{BBB}$ is actual problem in medicine [28]. The pharmacopuncture and acupuncture can be promising tool for brain drug delivery. Indeed, the pharmacopuncture at ST36 has been shown as the effective method for therapy of glioma and Parkinson's disease $[5,6,12]$. The electroacupuncture stimulation can cause the transient enhancement of the BBB permeability [13]. However, the mechanisms of pharmacopuncture and acupuncture-mediated effects on the brain physiology remain not fully understood.

In this pilot study, we analyzed the Evans Blue spearing after the dye injection into GB20 in the different time point (30 min for in vivo studies) and 3 hours (for ex vivo analysis). For in vivo studies, we choose a time of $30 \mathrm{~min}$ due to fast distribution of dye among head and its delivery to the meninges of the brain through PVSs of the trigeminal and olfactory areas. For ex vivo studies, we selected a time of 3 hours due to our previous results demonstrating that 3 hours are enough to deliver Evans Blue from the deep cervical lymph node into the meninges of the brain [29].

The pharmacopuncture at GB20 was selected due to the effective GB20 therapy of headache $[10,11]$. The GBmodulation of the $\mathrm{CBF}$ is considered as a possible mechanism underling the antinociceptive effects of GB stimulation [1618]. We hypothesized that pharmacopuncture at GB20 can be a tool for delivery of drugs into the brain via the drainage system of the brain. To test this hypothesis, we analyzed the distribution of Evans Blue in the mouse head and in the brain 30 min and 3 hours after the tracer injection at GB20 using in vivo and ex vivo the confocal microscopy and the histological analysis of the tested tissues. Our results revealed that the fast distribution of dye occurs through the trigeminal and olfactory areas (Fig. 1B, C). Long Evans Blue distribution involves the cribriform plate as the door for the delivery of dye into the brain (Fig. 2D, E). The cribriform plate is a main route of CSF outflow [30-35]. The cribriform plate is a fenestrated bony plate of the ethmoid bone that separates the cranial and nasal cavities. Once through the plate, CSF is absorbed by the lymphatic vessels in the nasal mucosa and drained into the cervical lymph nodes [36]. There has been speculation that the interstitial fluid and CSF leave the brain via the extracellular space between the olfactory sensory nerve axon bundles [37], as the intercellular space between axon bundles provide low-resistance directed pathways for fluid flow [38]. Taking into account these facts, we assume that the delivery of Evans Blue after its injection at GB20 can be through the cerebral fluid system connected with the cribriform plate. Our results confirmed our hypothesis and demonstrated the Evans Blue distribution in the brain tissues via PVSs (Fig. 3B-D). These data clearly suggest that pharmacopuncture at GB20 can be a promising tool for the perivascular delivery of drugs into the brain. Our results are consistent with those of others. Shetty and Zanirati [39] demonstrate that PVS is the perspective route of brain drug delivery. They discuss an important role of PVS in the passage of extracellular vesicles released from neural cells and the rapid targeting of the therapeutic vesicles into neural cells in the entire brain following an intranasal administration. Yang et al. [40] demonstrate the involvement of PVSs in the facial intradermal brain-targeted delivery.

In our pilot paper, we demonstrated the Evans Blue delivery into PVSs using only the injection of dye at GB20 that is limitation of this study. For the better understanding the effectiveness of pharmacopuncture at GB20 as an alternative method of brain drug delivery, further studies of pharmacopuncture should be performed in the same way on other acupuncture points to compare the effect difference.

\section{CONCLUSIONS}

In sum, the present study clearly demonstrates spearing of Evans Blue into the mouse head and in the brain after the dye injection at GB20. Our in vivo results revealed the fast distribution of dye in the mouse head via the trigeminal structures and the cribriform plate. In ex vivo data we observed delivery of Evans Blue into the brain tissues via PVSs of the main cerebral veins, such as the Superior sagittal sinus and the Transverse sinus and along the main cerebral arteries, including the Middle cerebral artery, the Circle of Willis, the Posterior cerebral artery, the Basilar artery. These results suggest that pharmacopuncture at GB20 can be a perspective tool for brain drug delivery via PVSs, which are 
considered as an important route for drug delivery into the brain [39].

\section{FUNDING}

This research was supported by RF Governmental Grant No. 075-15-2019-1885, Grant from RSF No. 20-15-00090; 2175-10088, Grant from RFBR 19-515-55016; China a, 20-01500308-a.

\section{AUTHORS' CONTRIBUTIONS}

O.S-G initiated and supervised this work. A.T., I.F., A.D., V.A. performed confocal analysis; N.A. performed the histological analysis; I.B., A.E., G.K., A.K., M.T., I.A., V.T., A.T. performed most of the experiments. O.S-G. and J.K. reviewed all results and wrote the manuscript. All authors were also involved in the results discussion and manuscript reviewing and editing.

\section{CONFLICT OF INTEREST}

The authors declare no conflict of interest.

\section{ORCID}

Oxana Semyachkina-Glushkovskaya, https://orcid.org/0000-0001-6753-7513

Inna Blokhina, https://orcid.org/0000-0003-1517-0857

Nikita Navolokin, https://orcid.org/0000-0001-7876-9758

Arina Evsukova, https://orcid.org/0000-0002-8725-8623

Georgy Karandin, https://orcid.org/0000-0001-6790-1565

Alexander Khorovodov,

https://orcid.org/0000-0002-5318-5200

Andrey Terskov, https://orcid.org/0000-0003-1111-9990

Ivan Fedosov, https://orcid.org/0000-0002-3619-245X

Alexander Dubrovsky, https://orcid.org/0000-0002-5897-9229

Maria Tzoy, https://orcid.org/0000-0002-4681-6465

Vasily Ageev, https://orcid.org/0000-0002-8264-9772

Ilana Agranovich, https://orcid.org/0000-0002-7903-3897

Valeria Telnova, https://orcid.org/0000-0001-8411-6366

Anna Tsven, https://orcid.org/0000-0002-9882-1354

Jürgen Kurths, https://orcid.org/0000-0002-5926-4276

\section{REFERENCES}

1. Hsieh CL. Acupuncture as treatment for nervous system diseases. Biomedicine (Taipei) 2012;2:51-7.

2. Kawakita K, Okada K. Acupuncture therapy: mechanism of action, efficacy, and safety: a potential intervention for psychogenic disorders? Biopsychosoc Med 2014;8:4.

3. Johansson BB, Haker E, von Arbin M, Britton $M$, Långström $G$, Terént A, et al. Acupuncture and transcutaneous nerve stimulation in stroke rehabilitation: a randomized, controlled trial. Stroke 2001;32:707-13.

4. Yu C, Wang L, Kong L, Shen F, Du Y, Kong L, et al. Acupoint combinations used for treatment of Alzheimer's disease: a data mining analysis. J Tradit Chin Med 2018;38:943-52.

5. Zhou J, Peng W, Xu M, Li W, Liu Z. The effectiveness and safety of acupuncture for patients with Alzheimer disease: a systematic review and meta-analysis of randomized controlled trials. Medicine (Baltimore) 2015;94:e933.

6. Song J, Lee SS, Lim S, Yeo S. Mechanism of the neuroprotective effect of injecting brain cells on ST36 in an animal model of Parkinson's disease. Neurosci Lett 2020;717:134698.

7. Cristian A, Katz M, Cutrone E, Walker RH. Evaluation of acupuncture in the treatment of Parkinson's disease: a doubleblind pilot study. Mov Disord 2005;20:1185-8.

8. Kloster R, Larsson PG, Lossius R, Nakken KO, Dahl R, XiuLing X, et al. The effect of acupuncture in chronic intractable epilepsy. Seizure 1999;8:170-4.

9. Kwon HJ, Kim JI, Lee MS, Choi JY, Kang S, Chung JY, et al. Acupuncture for sequelae of Bell's palsy: a randomized controlled trial protocol. Trials 2011;12:71.

10. Li Y, Zheng H, Witt CM, Roll S, Yu SG, Yan J, et al. Acupuncture for migraine prophylaxis: a randomized controlled trial. CMAJ 2012;184:401-10.

11. Liu L, Pei P, Zhao LP, Qu ZY, Zhu YP, Wang LP. Electroacupuncture pretreatment at GB20 exerts antinociceptive effects via peripheral and central serotonin mechanism in conscious migraine rats. Evid Based Complement Alternat Med 2016;2016: 1846296.

12. Soh KS. Hypothesis on the treatment of gliomas with acupuncture at the primo node corresponding to Zusanli (ST 36). Med Acupunct 2015;27:144-50.

13. Zhang S, Gong P, Zhang J, Mao X, Zhao Y, Wang H, et al. Specific frequency electroacupuncture stimulation transiently enhances the permeability of the blood-brain barrier and induces tight junction changes. Front Neurosci 2020;14:582324.

14. White A, Ernst E. A brief history of acupuncture. Rheumatology (Oxford) 2004;43:662-3.

15. Research Group of Acupuncture Anesthesia, Peking Medical College, Peking. Effect of acupuncture on pain threshold of human skin. Chin Med J 1973,86:35.

16. Yuan X, Hao X, Lai Z, Zhao H, Liu W. Effects of acupuncture at fengchi point (GB 20) on cerebral blood flow. J Tradit Chin Med 1998;18:102-5.

17. Im JW, Moon SK, Jung WS, Cho KH, Kim YS, Park TH, et al. Effects of acupuncture at GB20 on $\mathrm{CO} 2$ reactivity in the basilar and middle cerebral arteries during hypocapnia in healthy participants. J Altern Complement Med 2014;20:764-70. 
18. Byeon HS, Moon SK, Park SU, Jung WS, Park JM, Ko CN, et al. Effects of GV20 acupuncture on cerebral blood flow velocity of middle cerebral artery and anterior cerebral artery territories, and $\mathrm{CO} 2$ reactivity during hypocapnia in normal subjects. J Altern Complement Med 2011;17:219-24.

19. Sun BL, Wang LH, Yang T, Sun JY, Mao LL, Yang MF, et al. Lymphatic drainage system of the brain: a novel target for intervention of neurological diseases. Prog Neurobiol 2018;163164:118-43.

20. Wood H. Changes in brain drainage systems are linked to Parkinson disease. Nat Rev Neurol 2021;17:131.

21. Goadsby PJ, Knight YE, Hoskin KL, Butler P. Stimulation of an intracranial trigeminally-innervated structure selectively increases cerebral blood flow. Brain Res 1997;751:247-52.

22. Cheshire WP Jr, Wharen RE Jr. Trigeminal neuralgia in a patient with spontaneous intracranial hypotension. Headache 2009;49:770-3.

23. Turgut N, Unlü E, Hamamcioğlu MK, Güldiken B, Albayram S. Postural tremor as a manifestation of spontaneous intracranial hypotension. J Clin Neurosci 2010;17:255-7.

24. Mokri B. Spontaneous intracranial hypotension. Curr Pain Headache Rep 2001;5:284-91.

25. Podkovik S, Kashyap S, Bonda S, Bowen I, Calayag M. Spontaneous intracranial hypotension: case study and review of the literature. Cureus 2020;12:e7018.

26. Schindelin J, Arganda-Carreras I, Frise E, Kaynig V, Longair M, Pietzsch T, et al. Fiji: an open-source platform for biologicalimage analysis. Nat Methods 2012;9:676-82.

27. Lohrberg M, Wilting J. The lymphatic vascular system of the mouse head. Cell Tissue Res 2016;366:667-77.

28. Pardridge WM. CSF, blood-brain barrier, and brain drug delivery. Expert Opin Drug Deliv 2016;13:963-75.

29. Semyachkina-Glushkovskaya O, Fedosov I, Shirokov A, Vodovozova E, Alekseeva A, Khorovodov A, et al. Photomodulation of lymphatic delivery of liposomes to the brain bypas- sing the blood-brain barrier: new perspectives for glioma therapy. Nanophotonics 2021;10:3215-27.

30. Cserr HF, Knopf PM. Cervical lymphatics, the blood-brain barrier and the immunoreactivity of the brain: a new view. Immunol Today 1992;13:507-12.

31. Ma Q, Ries M, Decker Y, Müller A, Riner C, Bücker A, et al. Rapid lymphatic efflux limits cerebrospinal fluid flow to the brain. Acta Neuropathol 2019;137:151-65.

32. Weller RO, Kida S, Zhang ET. Pathways of fluid drainage from the brain--morphological aspects and immunological significance in rat and man. Brain Pathol 1992;2:277-84.

33. Kida S, Pantazis A, Weller RO. CSF drains directly from the subarachnoid space into nasal lymphatics in the rat. Anatomy, histology and immunological significance. Neuropathol Appl Neurobiol 1993;19:480-8.

34. Johnston M, Zakharov A, Papaiconomou C, Salmasi G, Armstrong D. Evidence of connections between cerebrospinal fluid and nasal lymphatic vessels in humans, non-human primates and other mammalian species. Cerebrospinal Fluid Res 2004;1:2.

35. de Leon MJ, Li Y, Okamura N, Tsui WH, Saint-Louis LA, Glodzik L, et al. Cerebrospinal fluid clearance in Alzheimer disease measured with dynamic PET. J Nucl Med 2017;58:1471-6.

36. Bradbury MW, Cole DF. The role of the lymphatic system in drainage of cerebrospinal fluid and aqueous humour. J Physiol 1980;299:353-65.

37. Szentistványi I, Patlak CS, Ellis RA, Cserr HF. Drainage of interstitial fluid from different regions of rat brain. Am J Physiol 1984;246(6 Pt 2):F835-44.

38. Syková E, Nicholson C. Diffusion in brain extracellular space. Physiol Rev 2008;88:1277-340.

39. Shetty AK, Zanirati G. The interstitial system of the brain in health and disease. Aging Dis 2020;11:200-11.

40. Yang W, Jin BH, Chen YJ, Cao C, Zhu JZ, Zhao YZ, et al. The involvement of perivascular spaces or tissues in the facial intradermal brain-targeted delivery. Drug Deliv 2019;26:393-403. 